\title{
A Longitudinal Study of the Relationships between Haemostatic, Lipid, and Oestradiol Changes during Nomal Human Pregnancy
}

\author{
Naveed Sattar ${ }^{1}$, lan A. Greer ${ }^{2}$, Ann Rumley ${ }^{3}$, Grace Stewart ${ }^{1}$, J ames Shepherd ${ }^{1}$, \\ Chris J. Packard ${ }^{1}$, Gordon D. O. Lowe ${ }^{3}$
}

From the University Departments of ${ }^{1}$ Pathologic al Bioc hemistry, $2 \mathrm{Obstetrics} \mathrm{and} \mathrm{Gynaecology,} \mathrm{and}$

${ }^{3}$ Medic ine, Royal Infimary, Glasgow, UK

\section{Summary}

Increased activation of both blood coagulation and fibrinolysis occurs during normal pregnancy. The responsible mechanisms are unclear, but may include increases in both oestradiol and blood lipids. We, therefore, studied the associations between fasting serum oestradiol, plasma cholesterol and triglyceride, and Factor VII activity, PAI activity, t-PA antigen, fibrin D-dimer, and vWF antigen in 10 women, each sampled on 6 occasions between 10 weeks and 35 weeks during normal pregnancy. Strong and similar individual correlations were observed between increases in FVII, PAI, t-PA and D-dimer (but not vWF) and increases in both oestradiol and triglyceride. Associations between increments in plasma cholesterol and haemostatic factors (except for FVII), were somewhat weaker. We, therefore, suggest that oestradiol-induced hypertriglyceridaemia may be a cause of elevations in plasma Factor VII activity, PAI and t-PA, and fibrin turnover (D-dimer) during normal pregnancy, but is poorly related to the increase in vWF antigen.

\section{Introduction}

Normal human pregnancy is associated with major increases in blood coagulation factors (fibrinogen, Factors VII, X and VIII and von Willebrand Factor [vWF]). Fibrinolytic factors (plasminogen activator inhibitor-1 (PAI-1), plasminogen activator inhibitor-2 (PAI-2), and tissue plasminogen activator (t-PA) antigen), and turnover of crosslinked fibrin, as measured by plasma fibrin D-dimer, are also increased (1-6). The physiological mechanisms underlying these changes are unclear, but they may be important for the maintenance of the placentouterine interface, or to meet the haemostatic challenge of delivery (1). The regulation of these changes is unclear. Possible mechanisms include (a) hormonal changes in pregnancy, particularly increased oestrogen which can induce changes in coagulation and fibrinolysis when administered exogenously in oral contraceptives (7) or hormone replacement therapy (HRT) (8); and (b) blood lipid changes in pregnancy, especially triglyceride which induces changes in coagulation and fibrinolysis following dietary intake (9) or oestradiol administration $(7,8)$. The objective of this study was to determine the associations between changes in fasting serum oestradiol, plasma triglyceride and cholesterol, and changes in Factor VII and PAI activities, and t-PA,

Correspondence to: Dr. Naveed Sattar, Department of Pathological Biochemistry, Macewen Building, Royal Infirmary NHS Trust, Glasgow G4 0SF, UK - Tel.: +44-141-211-4490; Fax-Number: +44-141-553-1703
D-dimer and vWF antigens; each of which has been related to risk of arterial and/or venous thrombosis in epidemiological studies. We have previously reported a detailed analysis of oestradiol, lipid and lipoprotein changes (and their inter-relationships) in this study (10).

\section{Subjects and Methods}

Twelve pregnant women who registered for obstetric care at Glasgow Royal Maternity Hospital were recruited. Eight were multipara with normal previous deliveries and four were primigravida. All women had no identifiable risk factors for the current pregnancy as determined by detailed obstetric history. During the course of pregnancy one woman emigrated, and another woman had a positive test for Down's syndrome in the fetus and was excluded by mutual consent. The remaining ten women all had normal course and outcome of pregnancy, term delivery, ate a customary diet and did not receive any medication known to interfere with lipid metabolism or lipid determination. Additionally, none were phenotype apo E2/E2, an inherited trait which may generate disturbances in the plasma lipid profile even in normolipaemic subjects. The subject characteristics are given in Table 1. The study was approved by the Research Ethics Committee of Glasgow Royal Infirmary University NHS Trust and each subject gave written informed consent.

\section{Experimental Design}

The pregnant women were first seen at 10 weeks gestation ( \pm 1 week) and thereafter at five week intervals until 35 weeks of pregnancy. Each woman was seen six times. The gestational age was confirmed by ultrasound. All subjects were sampled after an overnight fast of $12 \mathrm{~h}$. Twenty $\mathrm{ml}$ of blood was collected by venepuncture into $\mathrm{K}_{2}$ EDTA (10 $\mathrm{ml}$ - final concentration $1 \mathrm{mg} / \mathrm{ml}$ ), trisodium citrate $(5 \mathrm{ml}$ - final concentration $0.011 \mathrm{M})$, and plain tubes $(5 \mathrm{ml})$. Plasma and serum were harvested at $4^{\circ} \mathrm{C}$ by low speed centrifugation $(1000 \mathrm{~g})$ and aliquots of $\mathrm{K}_{2}$ EDTA plasma for lipid measurements and serum for oestradiol determination were used immediately. Citrated plasma aliquots for the determination of haemostatic factors were stored immediately at $-70^{\circ} \mathrm{C}$ until analysis.

\section{Laboratory Methods}

Plasma total cholesterol, triglyceride and HDL-cholesterol were determined by a modification of the standard Lipid Research Clinics Protocol (10). Oestradiol was determined by a quantitative immunoassay on the Technicon Immuno 1 system ("Heterogeneous Magnetic Separation Assay", Bayer Diagnostics, UK). FVIIc was determined by a one stage clotting assay in an ACL-300 Research coagulometer (IL, Warrington, UK). PAI activity was measured by a chromogenic assay (Chromogenix, Stockholm, Sweden). t-PA antigen (Biopool, Stockholm, Sweden), fibrin D-dimer (AGEN, Parsippany, NJ, USA), and vWF antigen (Dako, Copenhagen, Denmark) were all measured by ELISA techniques. 
Table 1 Clinical characteristics of study subjects $(\mathrm{n}=10)$

\begin{tabular}{lll}
\hline & Mean \pm SD & Range \\
\hline Age (yrs) & $29.5 \pm 4.0$ & $24-35$ \\
Height (cm) & $164 \pm 5$ & $155-173$ \\
Weight (Kg) & $62 \pm 6$ & $55-74$ \\
BMI (Kg/m²) & $22.9 \pm 2.3$ & $20-28$ \\
Blood pressure (mmHg)* & & \\
$\quad$ Systolic & $104 \pm 6$ & $95-110$ \\
$\quad \quad$ Diastolic & $69 \pm 4$ & $58-70$ \\
Length of gestation (wks) & $39.6 \pm 1.3$ & $38-41$ \\
Fetal Birth Weight (Kg) & $3.5 \pm 0.2$ & $3.1-3.8$ \\
\hline
\end{tabular}

*Blood pressure at booking

\section{Statistical Analysis}

The lipid, haemostatic and hormonal variables were parametrically distributed as judged by the examination of normal probability plots. Nevertheless, as numbers were small, data are presented as medians and ranges (Table 2).

Associations between changes in plasma lipid and haemostatic variables and oestradiol concentrations were tested by calculating the Pearson correlation coefficient $(\mathrm{R})$ and the coefficient of determination $\left(\mathrm{R}^{2}\right)$ which was expressed as a percentage, (i. e. $R^{2}$ gives the percentage of variation in the dependent variable which is explained by variation in the independent variable). The significance of association between pairs of variables was determined by linear regression. Values presented in Table 3 are the median (range) of the coefficients of determination (i.e median of 10 individual $\mathrm{R}^{2}$ values, one for each subject, where each $R^{2}$ value is based on 6 data points).

\section{Results}

Lipids

As pregnancy progressed from 10 to 35 weeks, median plasma triglyceride and cholesterol concentration rose from 0.78 to $2.55 \mathrm{mmol} / \mathrm{l}$, and from 4.40 to $7.20 \mathrm{mmol} / \mathrm{l}$, respectively (Table 2).

\section{Oestradiol}

From 10 weeks to 35 weeks of pregnancy median serum oestradiol concentration increased steadily from a median of $8.4 \mathrm{nmol} / \mathrm{l}$ to $73 \mathrm{nmol} / \mathrm{l}$.

\section{Haemostatic Parameters}

PAI activity increased by a factor of 2.56 from a median of $87 \%$ normal pool to $226 \%$ at 35 weeks. Increments in median circulating concentrations of t-PA (4.80-9.05 ng/ml), FVIIc (95.0-168.0 IU/dl), and vWF (136.0-231.0 IU/dl) were slightly less pronounced, whereas median D-dimer concentration increased by more than 4-fold ( 68.5 to $295.5 \mathrm{ng} / \mathrm{ml}$ ) (Table 2). The patterns of increase in plasma triglyceride, PAI activity, t-PA and D-dimer were similar, in that the maximum rise in each of these parameters occurred after 25 weeks (Table 2). In contrast, increments in FVIIc and vWF were more uniform in nature.

\section{Relationship between Serum Oestradiol, Plasma Triglyceride and Cholesterol, and Factor VII}

For each individual there were strong and significant relationships (Table 3 ) between the rise in factor VIIc and the increment in plasma triglyceride (Fig. 1a), and increment in serum oestradiol (Fig. 1b). Furthermore, the slopes of the association between increments in factor VIIc and plasma triglyceride or serum oestradiol were similar (3-fold vs 2.35-fold). The associations between rise in factor VIIc and increment in plasma cholesterol were equally strong (Table 3 ).

\section{Relationships between Serum Oestradiol, Plasma Triglyceride and Cholesterol, and PAI Activity}

For each individual there were strong and significant relationships (Table 3) between the rise in PAI activity, and the increment in plasma triglyceride (Fig. 2a), and increment in serum oestradiol (Fig. 2b). The associations between changes in plasma cholesterol and PAI activity were somewhat weaker (Table 3 ). In contrast to the pattern seen with FVII activity, however, the range of the slopes of association between increments in PAI activity and plasma triglyceride was greater than that between changes in plasma PAI activity and serum oestradiol (4.9 fold vs 2.6 fold).

\section{Relationship between Serum Oestradiol, Plasma Triglyceride and Cholesterol, and t-PA Antigen}

There were strong and significant relationships (Table 3) between the rise in t-PA antigen, and the increment in plasma triglyceride, and increment in serum oestradiol in 8 of the 10 individuals. Once again, the

\begin{tabular}{lllllll}
\hline Gestational age (wks) & 10 & 15 & 20 & 25 & 30 & 35 \\
\hline Triglyceride (mmol/l) & 0.78 & 1.20 & 1.40 & 1.53 & 1.73 & 2.55 \\
& $(0.6-1.55)$ & $(0.65-1.80)$ & $(0.80-2.30)$ & $(0.95-2.45)$ & $(1.25-2.75)$ & $(1.65-3.60)$ \\
Cholesterol (mmol/l) & 4.40 & 5.45 & 6.23 & 6.53 & 6.73 & 7.20 \\
& $(3.25-6.80)$ & $(4.00-7.50)$ & $(4.70-7.60)$ & $(5.05-8.55)$ & $(5.40-8.75)$ & $(5.60-8.45)$ \\
Oestradiol (nmol/l) & 8.4 & 19.0 & 38.7 & 46.2 & 58.1 & 73.0 \\
& $(3.6-22.6)$ & $(8.8-39.9)$ & $(17.6-60.4)$ & $(33.7-76.5)$ & $(40.7-73.6)$ & $(47.8-105.0)$ \\
FVIIc (IU/dl) & 95.0 & 121.0 & 125.5 & 132.0 & 151.0 & 168.0 \\
& $81.0-138.0$ & $99.0-137.0$ & $106.0-149.0$ & $107.0-179.0$ & $116.0-179.0$ & $137.0-193.0$ \\
PAI activity (\% pool) & 87.0 & 86.5 & 108.5 & 140 & 178.5 & 223.5 \\
& $33-125$ & $72-125$ & $59-153$ & $79-179$ & $129-222$ & $181-273$ \\
tPA-antigen (ng/ml) & 4.80 & 5.30 & 6.90 & 6.45 & 7.40 & 9.05 \\
& $2.40-6.50$ & $3.40-7.50$ & $4.70-7.60$ & $5.40-8.30$ & $5.00-10.00$ & $6.80-10.80$ \\
D-dimer (ng/ml) & 68.5 & 72.0 & 98.5 & 159.5 & 199.5 & 295.5 \\
& $29.0-127.0$ & $30.0-101.0$ & $47.0-123.0$ & $66.0-200.0$ & $127.0-310.0$ & $196.0-420.0$ \\
vWF (IU/dl) & 136.0 & 189.0 & 194.0 & 183.0 & 205.0 & 231.0 \\
& $93.0-253.0$ & $78.0-249.0$ & $81.0-252.0$ & $134.0-325.0$ & $89.0-296.0$ & $109.0-293.0$ \\
\hline
\end{tabular}

Table 2 Median (range) of lipid, oestradiol and haemostatic concentrations at each gestational time point for the 10 subjects 
associations between changes in plasma cholesterol and t-PA antigen were somewhat weaker (Table 3). Congruous to the association between the rise in PAI activity and the increment in plasma triglyceride, the relationship between the rise in t-PA antigen and the increment in plasma triglyceride also exhibited considerable variability between individuals in the slopes of association (4.7 fold difference, data not shown).

\section{Relationship between Serum Oestradiol, Plasma Triglyceride and Cholesterol, and D-dimer}

For each individual there was a strong and significant relationship (Table 3) between the rise in plasma D-dimer concentration and the increment in plasma triglyceride (Fig. 3a), whereas the association between alterations in plasma D-dimer and serum oestradiol concentrations were significant in 8 of the 10 subjects studied (Fig. 3b). The latter relationship appeared non-linear. Indeed, by utilising the logar-
Table 3 Associations in individual patients $(\mathrm{n}=10)$ between the change in plasma concentrations of haemostatic factors and alterations in plasma triglyceride and cholesterol and serum oestradiol concentrations

\begin{tabular}{llll}
\hline & Triglyceride & Cholesterol & Oestradiol \\
\hline FVII & $87.0(68.9-96.6)^{*}$ & $86.5(67.4-94.8)^{*}$ & $86.0(73.1-99.3)^{*}$ \\
PAI-1 & $86.7(77.0-97.6)^{*}$ & $67.9(41.3-92.8)$ & $83.5(65.6-97.1)^{*}$ \\
tPA & $89.2(51.1-97.3)^{\dagger}$ & $72.8(23.9-97.5)$ & $84.6(50.8-98.5)^{\dagger}$ \\
D-Dimer & $83.8(70.6-94.9)^{*}$ & $62.2(50.2-92.7)$ & $80.1(62.8-91.1)^{\dagger}$ \\
VWF & $39.8(0.10-88.1)$ & $51.5(5.4-80.4)$ & $45.7(9.5-89.2)$
\end{tabular}

Values presented are the median (range) of the coefficients of determination for the associations between the change in plasma concentrations of haemostatic factors and alterations in plasma triglyceride and cholesterol, and serum oestradiol concentrations.

$*$ p $<0.05$ in all 10 patients

$\dagger \mathrm{p}<0.05$ in 8 of the 10 patients.

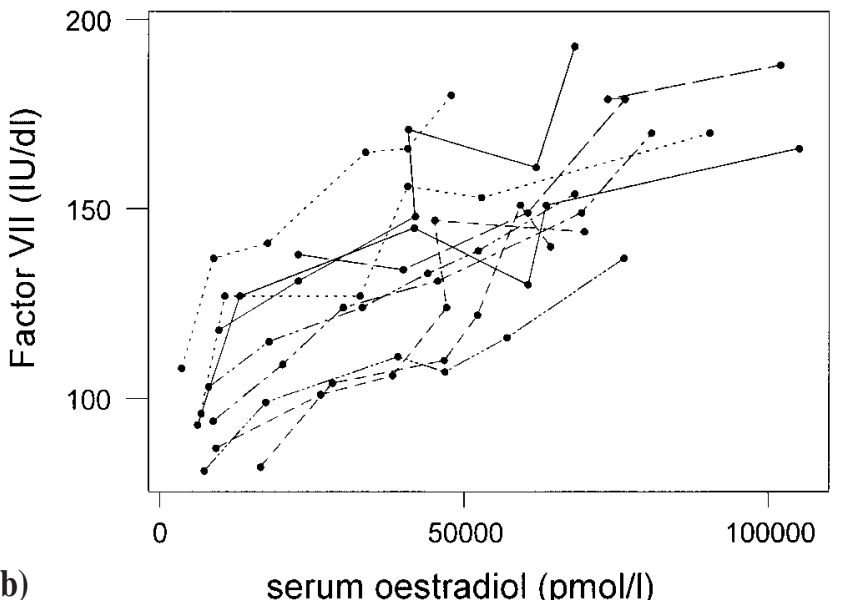

a)

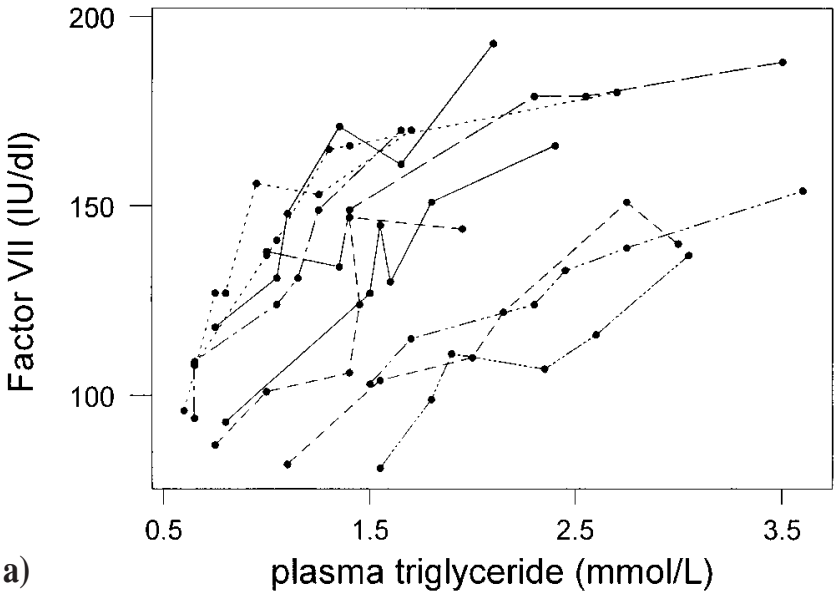

b)

Fig. 1 a) This graph demonstrates the sequential relationship between plasma triglyceride (mmol/l) and FVIIc in each of the 10 subjects as pregnancy progressed. Lines connect the data for a given women. A significant and strong linear correlation (Table 3) between increment in plasma triglyceride and FVIIc was observed in each of the individuals studied. $b$ ) This graph demonstrates the sequential relationship between serum oestradiol (mmol/l) and factor VIIc in each of the 10 subjects as pregnancy progressed. Lines connect the data for a given women. A significant and strong linear correlation (Table 3 ) between increments in the two parameters was observed in each of the individuals studied
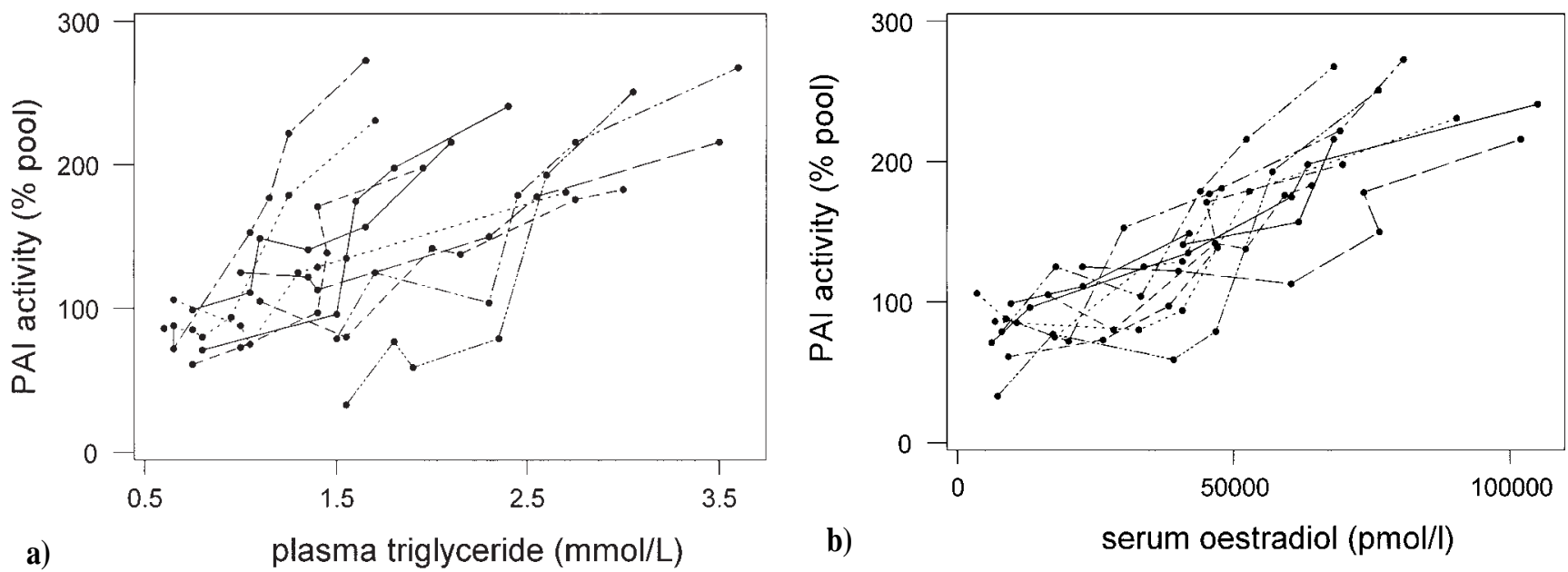

Fig. 2 a) This graph demonstrates the sequential relationship between plasma triglyceride (mmol/l) and PAI-1 activity in each of the 10 subjects as pregnancy progressed. Lines connect the data for a given women. A significant and strong linear correlation (Table 3) between increments in the two parameters was observed in each of the individuals studied, although the slope of the associations appeared to vary markedly ( 4.9 fold difference). $b$ ) This graph demonstrates the sequential relationship between serum oestradiol (mmol/l) and PAI-1 activity in each of the 10 subjects as pregnancy progressed. Lines connect the data for a given women. A significant and strong linear correlation (Table 3) between increments in the two parameters was observed in each of the individuals studied 


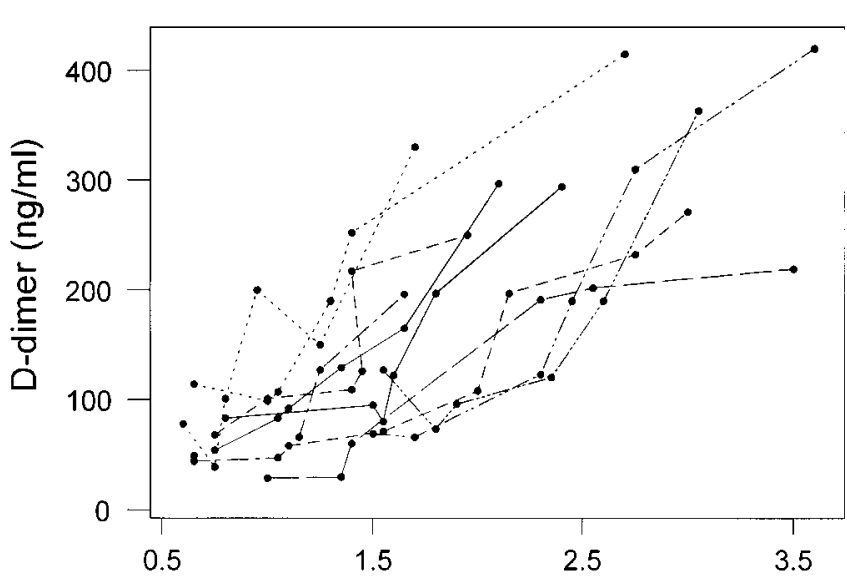

a)

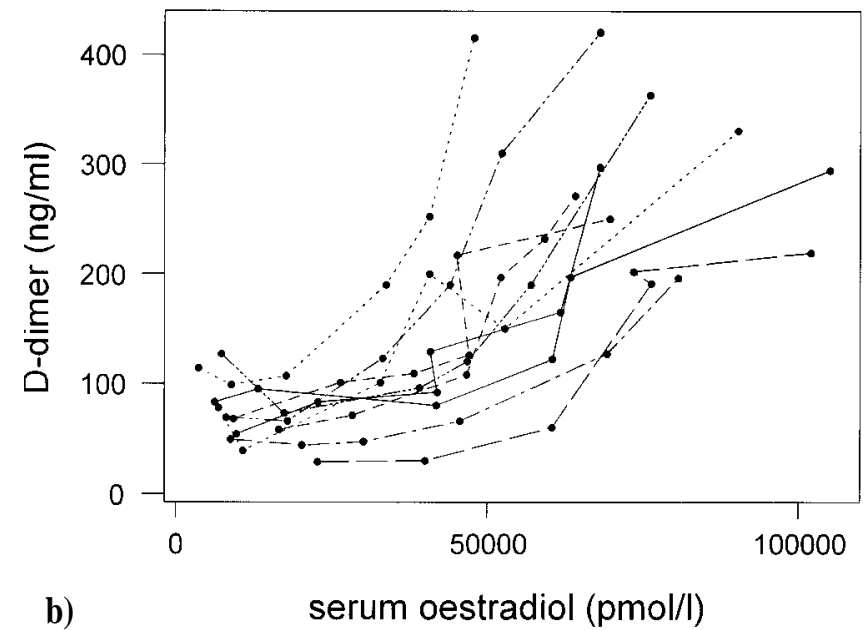

b) ithm of D-dimer, the median regression for the association between changes in oestradiol and D-dimer improved from $80.1 \%$ (linear) to $86.5 \%$ (logarithmic). However, this improvement was not statistically significant.

\section{Relationship between Serum Oestradiol, Plasma Triglyceride and Cholesterol and $v W F$}

The associations between changes in vWF with increases in serum oestradiol, plasma triglyceride or cholesterol, in each individual, were considerably weaker than the associations described above (Table 3).

\section{Disc ussion}

The increases in coagulation and fibrinolytic variables during normal pregnancy which were observed in the present study were similar to those described in previous studies (1-6), as were the increases in serum oestradiol and plasma lipids (10-12). To our knowledge, the present study is the first to report strong and similar correlations in individual patients between the increments in Factor VII and PAI activities, t-PA and D-dimer antigens, and serum oestradiol and plasma triglyceride. These results are consistent with the hypothesis that oestradiol-induced hypertriglyceridaemia may be one mechanism through which increases in these coagulation and fibrinolytic variables occur during pregnancy.

There is much evidence that hypertriglyceridaemia in non-pregnant subjects is associated with increases in Factor VII and PAI activities: in part, this may reflect causal effects of triglyceride-rich lipoproteins on both activation of Factor VII, and synthesis and release of PAI-1 from endothelial cells (9-13). Exogenous oral oestrogens increase Factor VII activity even in low doses (HRT) $(7,8)$, and it has been suggested that this may be related to increased plasma triglyceride levels (14). While lower doses of exogenous oestrogens (HRT and contraceptives) decrease plasma levels of PAI-1 (7, 8, 14), pregnancy results in far higher levels of endogenous oestradiol. Thus a stimulatory effect on PAI-1 in pregnancy cannot be excluded, and further research is required to examine the effects of varying oestradiol doses on PAI-1 synthesis in vitro. It should be noted that the assay of PAI activity used in the present study is specific for PAI-1, and insensitive to PAI-2. While both PAI-1 and PAI-2 increase greatly during pregnancy, only PAI-1 reacts sufficiently rapidly to have a major role in the regulation of plasma t-PA activity $(6,15)$. It should also be noted that the relationship between PAI-1 and triglyceride may be related to insulin resistance (13).

The increase in plasma t-PA antigen during pregnancy was only slightly lower than the increase in PAI activity. This may reflect a primary increase in PAI which binds t-PA, and circulates as t-PA-PA1 complexes. These complexes are detected by the t-PA assay used in the present study. Additionally, or alternatively, it may reflect endothelial release of t-PA. While the increase in t-PA antigen during pregnancy was accompanied by a similar increase in vWF antigen, suggesting a related change in endothelial release, the correlation between the t-PA increment and the oestradiol and triglyceride increments was much stronger than the correlation between the vWF increment and the oestradiol and triglyceride increments. This finding suggests that either oestradiol-induced hypertriglyceridaemia is more important in endothelial release of t-PA than vWF in normal pregnancy, or that the increase in t-PA may be secondary to a primary increase in PAI-1 due to oestradiol-induced hypertriglyceridaemia.

The correlations between increases in plasma fibrin D-dimer, oestradiol and triglyceride during normal pregnancy suggest that the changes in Factor VII associated with oestradiol-induced hypertriglyceridaemia may result in increased fibrin turnover in vivo. There is evidence in non-pregnant subjects that increasing Factor VII activity is associated with increased thrombin activity in vivo, as measured by plasma levels of fibrinopeptides, fragments F1+2, or thrombin-antithrombin complexes $(16,17)$. Further studies are required to examine the relationships between oestradiol, triglyceride, Factor VII and D-dimer in larger number of individuals.

These findings may be relevant not only to the possible mechanisms of change in coagulation and fibrinolysis during pregnancy, but also to the pathogenesis of atherosclerosis and thrombosis. Pregnancy is an interesting transient model of hyperlipidaemia and the development of an "atherogenic" lipoprotein profile (12) is also related to changes in 
coagulation and fibrinolysis, which have been related to thrombotic risk in epidemiological studies $(17,18)$. A notable finding in the present study was the inter-individual variability in increments in coagulation and fibrinolytic variables with elevations in oestradiol and triglycerides during pregnancy, suggesting the possibility of gene-environmental interactions (19). We suggest that further studies of individual changes in hormones, lipids and haemostatic variables during pregnancy may offer insights into the regulation of coagulation and fibrinolysis, and their relationships to atherothrombosis.

In conclusion, we have shown that in individual women undergoing normal pregnancy, there are significant correlations between oestradiol, triglyceride, Factor VII, PAI, t-PA and fibrin D-dimers, but not vWF, suggesting that oestradiol induced triglyceride changes are responsible these changes in coagulation and fibrinolysis.

\section{Acknowledgements}

This work was supported by grants from the Medical Research Council/CSO (SOHHD) Research grant (No. G9500819) and the Scottish Office Home and Health Department (Grant No. K/MRS/50/C2256).

\section{References}

1. Greer IA. Haemostasis and thrombosis in pregnancy. In: Bloom AL, Forbes CD, Thomas DP, Tuddenham EGD (eds). Haemostasis and Thrombosis, 3rd edn. Edinburgh: Churchill Livingstone 1994: 987-1016.

2. Hellgren M, Blombäck M. Studies on blood coagulation and fibrinolysis in pregnancy, during delivery and in the puerperium. 1. Normal condition. Gyn Obstet Invest 1981; 12: 141-54.

3. Beller FK, Ebert $\mathrm{C}$. The coagulation and fibrinolytic enzyme systems in normal pregnancy and the puerperium. Europ J Obstet Gynecol Repro Biol 1982; 13: 177-97.

4. Stirling Y, Woolf L, North WRS, Seghatchian MJ, Meade TW. Haemostasis in normal pregnancy. Thromb Haemost 1984; 52: 176-82.

5. Ballageer V, Mombaerts P, Declerk PJ et al. Fibrinolytic response to venous occlusion and fibrin fragment D-dimer levels in normal and complicated pregnancy. Thromb Haemost 1987; 58: 1030-2.

6. Kruithof EKO, Chien TT, Gudinchet A et al. Fibrinolysis in pregnancy: a study of plasminogen activation inhibitors. Blood 1987; 69: 460-6.
7. Kluft C, Lansink M. Effects of oral contraceptives on haemostasis variables. Thromb Haemost 1997; 78: 315-26.

8. Meade TW. Hormone replacement therapy and haemostatic function. Thromb Haemost 1997; 78: 765-9.

9. Mitropoulos KA. Lipid-thrombosis interface. Br Med Bull 1994; 50: 813-32.

10. Sattar N, Greer IA, Louden J et al. Lipoprotein subfraction changes in normal pregnancy: threshold effect of plasma triglyceride on appearance of small, dense low density lipoprotein. J Clin Endocrinol Metab 1997; 82: 2483-91.

11. Montelongo A, Lasuncion MA, Pallardo LF, Herrara E. Longitudinal study of plasma lipoproteins and hormones during pregnancy in normal and diabetic women. Diabetes 1992; 42: 1651-9.

12. Silliman K, Shore V, Forte TM. Hypertriglyceridaemia during late pregnancy is associated with the formation of small dense low-density lipoproteins and the presence of large buoyant high-density lipoproteins. Metabolism 1994; 43: 1035-41.

13. Juhan-Vague I, Alessi MC. Plasminogen activation inhibitor 1 and atherothrombosis. Thromb Haemost 1993; 70: 138-43.

14. Lowe GDO. Coagulation, fibrinolysis and hormone replacement therapy. In: Shaw RW (ed). Oestrogen deficiency. Causes and consequences. Carnforth, UK: Parthenon 1996; 29-44.

15. Thorsen S, Philips M, Selmer J, Lecander I, Astedt B. Kinetics of inhibition of tissue-type and urokinase-type plasminogen activator by plasminogenactivator inhibitor type 1 and type 2. Eur J Biochem 1988; 175: 33-9.

16. Miller GJ. Lipids and Coagulation. In : Poller L, Ludlam CA (eds). Recent advances in blood coagulation, 7. Edinburgh: Churchill Livingstone 1997; $125-40$.

17. Lowe GDO. Haemostatic risk factors for arterial and venous thrombosis. In: Poller L, Ludlam CA (eds). Recent advances in Blood Coagulation, 7. Edinburgh: Churchill Livingstone 1997; 69-96.

18. Lowe GDO, Rumley A, Woodward M, Morrison CE, Phillippon H, Lane DA, Tunstall-Pedoe H. Epidemiology of coagulation factors, inhibitors and activation markers: the third Glasgow MONICA survey I. Illustrative reference ranges by age, sex and hormone use. Br J Haematol 1997; 97 : 775-84.

19. Green F, Humphries S. Genetic determinants of arterial thrombosis. Ballieres Clin Haematol 1994; 7: 675-92.

Received February 25, 1998 Accepted after resubmission September 9, 1998 